\title{
Safety and efficacy of cilostazol in the management of intermittent claudication
}

\author{
Yung-Wei Chi \\ Carl J Lavie \\ Richard V Milani \\ Christopher J White \\ Department of Cardiology, Ochsner \\ Clinic Foundation, New Orleans, LA, \\ USA
}

\begin{abstract}
Peripheral arterial disease (PAD) is a major health problem affecting millions of patients worldwide. Many will suffer from intermittent claudication (IC), which leads to marked impairment of quality of life (QoL). Besides surgical and endovascular interventions to improve limb-specific outcomes, pharmacotherapy is an effective tool in the treatment of IC. Cilostazol, a Federal Drug Administration-approved medication for the treatment of IC, has demonstrated consistent efficacy in improving exercise capacity and overall health-related QoL. This manuscript will review the pharmacokinetics, safety, and efficacy of cilostazol in the treatment of patients with IC as well as compare this agent with other proven non-invasive therapies for PAD.
\end{abstract}

Keywords: peripheral arterial disease, cilostazol, intermittent claudication, supervised exercise therapy

\section{Introduction}

Peripheral arterial disease (PAD) is most commonly due to atherosclerosis, affects millions of patients worldwide, and is associated with significant morbidity and mortality (Hiatt 2001). PAD is defined as an obstruction of the infra-renal abdominal aorta and lower extremity arteries that reduces arterial flow during exercise and/or at rest. Intermittent claudication (IC) is the most common symptom in patients with PAD and is associated with exercise-induced discomfort in the muscles, relieved with rest; this may lead to marked impairment of quality of life $(\mathrm{QoL})$ and daily activities. However, IC is one symptom. Many patients have limited walking distance (atypical claudication) but do deny IC, ie, fatigue or tired or aching legs. Investigators have found that peak walking distance, peak walking time, and peak heart rate are all significantly reduced in PAD patients versus healthy controls (Hiatt et al 1992).

Following the general principle that more intense activities require greater oxygen consumption, the requirement for oxygen consumption in a healthy person increases from $4 \mathrm{~mL} / \mathrm{kg} / \mathrm{min}$ at rest to approximately $7 \mathrm{~mL} / \mathrm{kg} / \mathrm{min}$ for self-care, $9 \mathrm{~mL} / \mathrm{kg} / \mathrm{min}$ for housecleaning, $13 \mathrm{~mL} / \mathrm{kg} / \mathrm{min}$ for dancing, $14 \mathrm{~mL} / \mathrm{kg} / \mathrm{min}$ for golf, $24 \mathrm{~mL} / \mathrm{kg} / \mathrm{min}$ for jogging, and $31 \mathrm{~mL} / \mathrm{kg} / \mathrm{min}$ for marathon running (Ainsworth et al 2000). Considering that the peak oxygen capacity for an individual with PAD rarely exceeds $14.8 \pm 0.8 \mathrm{~mL} / \mathrm{kg} / \mathrm{min}$ (Hiatt et al 1992), it appears that even when working at maximum capacity, many PAD patients may lack the ability to complete the activities of daily life. Subjects with IC exhibited a mean exercise duration of 10.6 minutes, which was approximately half that demonstrated by age-matched controls $(\mathrm{p}<0.05)$ (Hiatt et al 1987). In addition, the PAD-associated deficits in exercise performance were accompanied by a roughly $50 \%$ decline in oxygen capacity, indicating that the level of impairment in PAD is comparable to that associated with class III congestive heart failure (Hiatt 2001).

A number of drugs (Table 1) have been tested for IC therapy, with mostly disappointing results. Among the multitude of failed pharmacotherapies, prostaglandins 
appeared to have promising potential more than a decade ago but more recently have been shown to have only modest efficacy (Hiatt 2001). Antiplatelet medications, serotonin blockers, and vasodilators have also been studied, but thus far none have demonstrated significant benefits for patients with IC (Hiatt 2002). In the US, two agents have been approved for such an indication (cilostazol and pentoxifylline), but only cilostazol has demonstrated consistent efficacy in both extending exercise capacity and improving QoL (Beebe et al 1999; Dawson et al 2000; Money et al 1998). As a result, cilostazol may be the most clinically effective pharmacologic option for IC in US patients. This manuscript will provide an overview of the pharmacology, metabolism, safety, and efficacy of cilostazol in the treatment of patients with typical IC and will compare this agent to other proven, non-invasive therapies for PAD.

\section{Pharmacology}

Cilostazol (Figure 1) was approved by the Federal Drug Administration (FDA) in 1999 for the treatment of IC (Kumar and Bhattacharya 2007). It became available in generic form in 2006. It is a reversible selective inhibitor of phosphodiesterase (PDE) type III. Similar to other members of its class, one of the primary effects of cilostazol is an increase in cyclic adenosine monophosphate (cAMP) in platelets, vascular smooth muscle, endothelial cells, and other PDE-III-rich cells, which may lead to a number of beneficial outcomes (Table 2). Among these potential benefits, cilostazol has been shown to inhibit platelet activation/aggregation, reduce thrombosis, enhance vasodilation (Chapman and Goa 2003), and induce nitric oxide (NO) production (Hashimoto et al 2006) as well as inhibit smooth muscle cell proliferation (Takahashi et al 1992; Hayashi et al 2000), increase limb blood flow (Elam et al 1998), increase plasma high-density

Table I Pharmacotherapies tested for intermittent claudication therapy
- Prostaglandin analogs
- Antiplatelets
- Serotonin blockers
- Vasodilators
- Naftidrofuryl
- L-Arginine
- Levocarnitine and propionyl levocarnitine
- Immunomodulation therapy
- Antimicrobial therapy
- Phosphodiesterase inhibitors

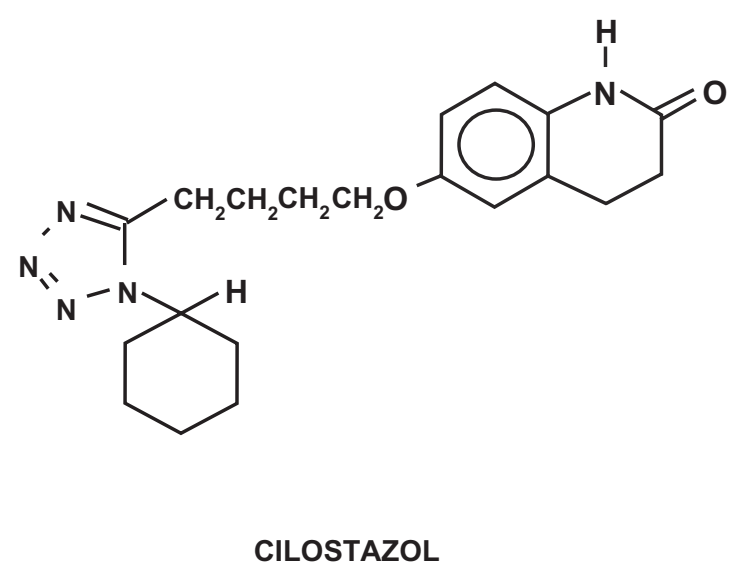

Figure I Cilostazol biochemical composition.

lipoprotein-cholesterol (HDL-C) (Lee et al 2001) and reduce plasma triglyceride levels (Elam et al 1998), potentiate angiogenesis (Lee et al 2001), and reduce inflammation (Agrawal et al 2007). Although the exact mechanism for cilostazol's benefits in IC remains unknown, any of these properties could potentially contribute to the symptomatic efficacy associated with this pharmacologic agent.

In vitro and/or ex vivo studies using platelets from healthy volunteers, in patients with atherosclerosis and in patients with cerebrovascular disease, have shown that cilostazol reversibly inhibits the primary and secondary stages of platelet aggregation (Chapman and Goa 2003). Cilostazol has also been found to be superior to aspirin in suppressing platelet aggregation ex vivo and to have some effects on platelet function more potent than the antiplatelet drug ticlopidine (Ikeda et al 1987). Cilostazol has been shown to reduce platelet aggregation without prolonging bleeding time (Tamai et al 1999). While selectively inhibiting the function of human platelets, cilostazol does not affect the production of prostacyclin and does not inhibit the function of vascular endothelial cells (Tani et al 1992). Cilostazol also produces vasodilatation by inhibiting smooth muscle cell contraction secondary to the elevation of cAMP levels and the blocking of calcium ion release (Chapman and Goa 2003). Cilostazol has also been shown to induce NO production by activating endothelial nitric oxide synthase (eNOS) (Hashimoto et al 2006).

Cilostazol has been shown to inhibit neointimal hyperplasia and restore endothelial function after balloon injury to the carotid artery of rats by inhibiting vascular smooth muscle cell growth, thus stimulating the production of hepatocyte growth factor in rapidly regenerating endothelial cells (Aoki et al 2001). 
Table 2 Beneficial effects of cilostazol

- Inhibits platelet activation and aggregation

- Reduces thrombosis

- Inhibits smooth muscle cell contraction

- Inhibits smooth muscle cell proliferation

- Increases limb blood flow

- Increases plasma high density lipoprotein-cholesterol levels

- Reduces plasma triglyceride levels

- Potentiates angiogenesis

- Reduces inflammation

Cilostazol also has beneficial effects on lipids. After 12 weeks of therapy with cilostazol, a $10 \%$ increase in HDL-C and a $15 \%$ decrease in plasma triglycerides have been noted (Elam et al 1998; Lee et al 2001). These beneficial changes with cilostazol were evident at 2 weeks for HDL-C and 4 weeks for triglycerides and persisted throughout the 12 weeks of therapy. There were no significant effects on low-density lipoprotein-cholesterol (LDL-C) or total cholesterol, but there was an increase of $6 \%$ in apolipoprotein A levels (Elam et al 1998, Lee et al 2001). More interestingly, in animal models of myocardial infarction (MI), cilostazol in combination with the potent statin atorvastatin had synergistic effects on nitric oxide production and reduction of MI size (Manickavasagam et al 2007). Cilostazol may potentiate angiogenesis and has been shown to increase vascular endothelial growth factors levels, which were significantly related to improved levels of exercise tolerance (Lee et al 2001).

Additionally, in hypertensive patients with type 2 diabetes mellitus, inflammatory markers - such as highly-sensitive $\mathrm{C}$ reactive protein (hs-CRP), erythrocyte sedimentation rate (ESR), total leukocyte count, and plasma malondialdehyde - have been shown to be elevated, suggesting high levels of inflammation and oxidative stress. Cilostazol has been shown to reduce these inflammatory markers (Agrawal et al 2007). In a randomized, open, add-on preventive controlled trial specifically assessing the effects of cilostazol on inflammatory markers, 60 hypertensive patients $\geq 45$ years of age with diabetes mellitus were recruited. Cilostazol $100 \mathrm{mg} 2 \times /$ day was given to 30 patients in addition to their standard medical treatment. At the 1-month follow-up, the cilostazol group showed significant $(\mathrm{p}<0.001)$ reductions in hs-CRP (24\%), ESR (39\%), total leukocyte count $(13 \%)$, and plasma malondialdehyde (18\%).

\section{Metabolism}

Cilostazol is taken orally, and maximal plasma concentration of the drug is observed at 2.7 hours after the final administration, reaching its steady state within 96 hours (Bramer et al 1999). Cilostazol is highly bound to plasma proteins, primarily albumin, and has a free fraction of up to $5 \%$ in patients with IC (Bramer et al 1999). It is metabolized by the hepatic enzyme CYP3A4 and to a lesser extent CYP2C19, primarily by oxidative metabolism to numerous metabolites (Bramer et al 1997). The main metabolites identified include dehydro-cilostazol and monohydroxy-cilostazol, which have inhibitory effects on platelet aggregation (Bramer et al 1997).

\section{Safety}

A black box warning indicates that cilostazol should be avoided in patients with congestive heart failure. The warning does not specify the heart failure classification or the degree of severity that should be avoided. This warning was imposed by the FDA because of the prior experience of chronic oral milrinone therapy (another PDE-III inhibitor) in patients with heart failure (Packer et al 1991). Regarding cardiovascular events and mortality, the risks associated with cilostazol seem to be numerically comparable to placebo. In an analysis that included the entire cilostazol safety database in addition to 4 trials conducted outside the US (Pratt 2001), no significant differences were found between cilostazol- and placebo-treated groups in rates of MI, stroke, or cardiovascular death during follow-up (Pratt 2001). Moreover, in two trials the distribution of total cardiovascular morbidity and mortality was actually higher in the placebo group $(8 \%)$ than among patients receiving either $50 \mathrm{mg} 2 \times /$ day ( $6 \%$ ) or $100 \mathrm{mg} 2 \times /$ day (7\%) doses of cilostazol (Pratt 2001). Thus, cilostazol seems to be a relatively safe alternative for IC therapy, offering an acceptable risk-benefit profile for patients with this condition. However, because of the existing black box warning against giving cilostazol to patients with congestive heart failure, the authors do not recommend prescribing cilostazol to such patients with IC.

Similar to other drugs in its class, cilostazol is associated with a fairly high frequency of non-life threatening adverse effects, particularly headaches, which may occur as a result of its vasodilatory properties. Headaches occurred in 443 of 1374 patients $(32 \%)$ treated with cilostazol compared with 40 of $355(11 \%)$ treated with the other approved therapy for IC, pentoxifylline, and 127 of 973 (13\%) treated with placebo (Pratt 2001). In addition, more patients in the cilostazol group than in the pentoxifylline and placebo groups reported diarrhea ( $17 \%$ versus $8 \%$ and $7 \%$, respectively), abnormal stools (14\% versus $5 \%$ and $4 \%$, respectively), peripheral edema ( $7 \%$ versus $4 \%$ and $4 \%$, respectively), and palpitations ( $9 \%$ versus $2 \%$ and $1 \%$, respectively). 
A large $(n=1439)$ ongoing, long-term, post-marketing clinical study conducted in the US, Japan and other Asian countries, South America, and the UK from 1999 through 2003 recently concluded (Hiatt 2006). Patients exposed to marketed formulations of cilostazol were evaluated to measure spontaneously reported adverse events. At the end of the study, 872 treatment-emergent adverse events were reported. A total of 773 events were considered serious and 99 non-serious according to FDA definitions. Of these, 61 events in 41 cases were considered drug related by the study investigators. Treatment-related serious adverse events occurring with the greatest frequency included congestive heart failure (14 cases; $2 \%$ ), gastrointestinal hemorrhage ( 6 cases; $1 \%$ ), atrial fibrillation ( 4 cases; $<1 \%$ ), diarrhea ( 3 cases; $<1 \%$ ), and dyspnea ( 3 cases; $<1 \%$ ). At total of 572 events were considered unexpected. Of the 872 serious and non-serious events observed, 181 required the discontinuation of cilostazol due to adverse events. The most frequent adverse events requiring discontinuation were congestive heart failure (26 cases; $2 \%$ ), diarrhea (15 cases; $1 \%$ ), and headaches (14 cases; $1 \%)$.

\section{Efficacy}

Once the goals of risk factor modification for patients with PAD have been met, the focus of PAD management shifts from general cardio-protection to treatment strategies specific for the clinical manifestations of PAD such as IC. There are consistent data supporting the efficacy of cilostazol for IC. In one the of most important studies included in the agent's New Drug Application, 698 patients with moderate to severe IC were randomized to a standard dose of cilostazol $100 \mathrm{mg} 2 \times /$ day, a standard dose of pentoxi-

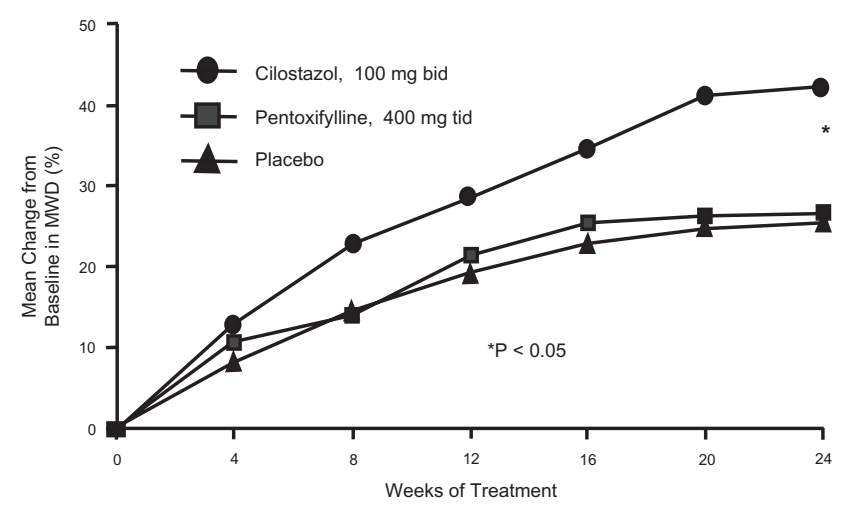

Figure 2 Mean percent change in maximal walking distance over time among intermittent claudication patients randomized to cilostazol $100 \mathrm{mg} 2 \times /$ day ( $n=227)$, pentoxifylline $400 \mathrm{mg} 3 \times /$ day $(n=232)$, or placebo $(n=239)$ for 24 weeks. $\mathrm{MWD}=$ maximal walking distance. Reprinted from Dawson, DL, Cutler BS, HiattWR, et al 2000.A comparison of cilostazol and pentoxifylline for treating intermittent claudication. Am J Med, 109:523-30. Copyright @ 2000, with permission from Elsevier. fylline (400 mg $3 \times /$ day), or placebo ( $3 \times /$ day) for a period of 24 weeks. By the end of the study, patients included in the cilostazol group had shown a 54\% improvement in maximal walking distance from the baseline compared with increases of $30 \%$ in the pentoxifylline group $(\mathrm{p}<0.05)$ and $34 \%$ in the placebo group $(\mathrm{p}<0.05)$ (Figure 2$)$ (Dawson et al 2000). Earlier trials have demonstrated a dose-response relationship with cilostazol. In one study (Beebe et al 1999), IC patients who received cilostazol $100 \mathrm{mg} 2 \times /$ day for 24 weeks increased their maximal walking distance by $51 \%$ over the baseline, whereas those who received a $50 \mathrm{mg} 2 \times$ /day dose showed an increase of only $38 \%$ compared to baseline values ( $\mathrm{p}<0.001$ for both doses versus placebo) (Figure 3). In a second trial, the net improvement in maximal walking distance over placebo was $21 \%$ with cilostazol $100 \mathrm{mg} 2 \times /$ day compared to $7 \%$ for cilostazol $50 \mathrm{mg} 2 \times /$ day. Notably, patients in both the $50 \mathrm{mg}$ and $100 \mathrm{mg}$ cilostazol groups showed improvements in global measures of physical functioning compared to placebo-treated patients $(\mathrm{p}<0.05)$ (Strandness et al 1998). Collectively, more than 2,700 IC patients were investigated in eight clinical trials with variable results, but there was a consistent trend toward improvement with cilostazol over placebo. Dawson et al (1998) and Money et al (1998) have shown a significant improvement in maximal walking distance in those who received cilostazol $100 \mathrm{mg} 2 \times /$ day compared with those who received placebo. Recently, a meta-analysis from six phase III cilostazol trials (Regensteiner et al 2002) with study durations of 12 to 24 weeks found a net benefit of the drug in both the constant workload treadmill and graded treadmill tests. Among the 749 patients who had been evaluated with a constant workload treadmill test, cilostazol $100 \mathrm{mg}$ $2 \times /$ day was associated with a $76 \%$ improvement in maximal walking distance from the baseline compared with a $20 \%$ improvement for placebo $(\mathrm{p}<0.0001)$. Similarly, 895 patients evaluated with a graded treadmill test showed a $40 \%$ maximal walking distance improvement with cilostazol $100 \mathrm{mg} 2 \times$ /day compared with a $20 \%$ increase with placebo $(\mathrm{p}<0.05)$ (Figure 4$)$. When data for both types of treadmill protocols were combined, cilostazol $100 \mathrm{mg}$ $2 \times /$ day demonstrated superiority over placebo in both maximal walking distance $(\mathrm{p}<0.05)$ and pain-free walking distance values $(\mathrm{p}<0.05)$. Furthermore, cilostazol demonstrated efficacy on several pain and physical dimensions on the Short Form-36 physical functioning scores and Walking Impairment Questionnaire, indicating that the drug's beneficial effects on exercise capacity are indeed 


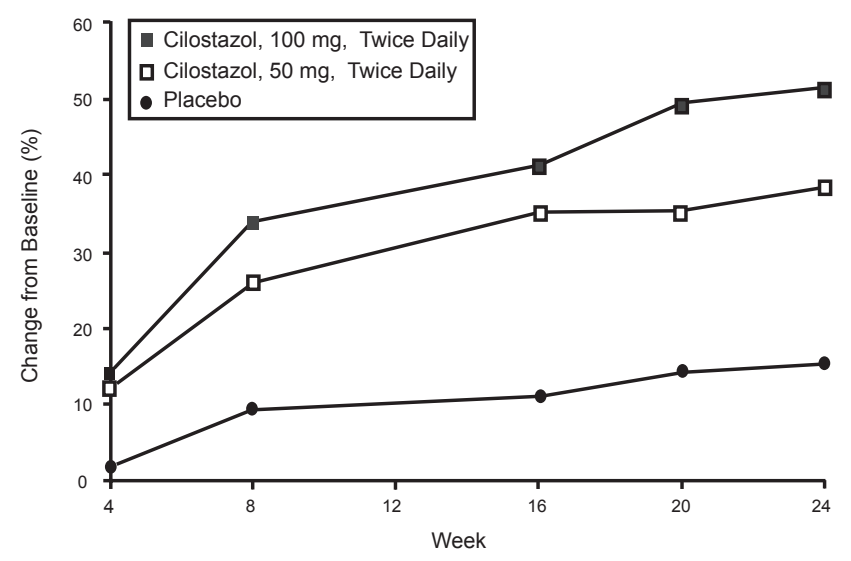

Figure 3 Mean percent change in maximal walking distance (MWD) among intermittent claudication patients receiving cilostazol $100 \mathrm{mg} 2 \times /$ day $(n=140)$, cilostazol $50 \mathrm{mg} 2 \times /$ day $(n=139)$, or placebo $(n=140)$ for 24 weeks. Reprinted from Beebe, HG, Dawson DL, Cutler BS, et al 1999. A new pharmacological treatment for intermittent claudication: results of a randomized, multicenter trial. Arch Intern Med, I59:204I-50. Copyright (C) 1999 with permission from American Medical Association.

accompanied by meaningful improvements in QoL. The beneficial effects of cilostazol were statistically superior to placebo as early as 4 weeks following treatment onset. However, the therapeutic effects of cilostazol were seen to improve progressively as study duration increased. In clinical practice, 12 to 24 weeks should be expected as a typical waiting period for improvement to occur.

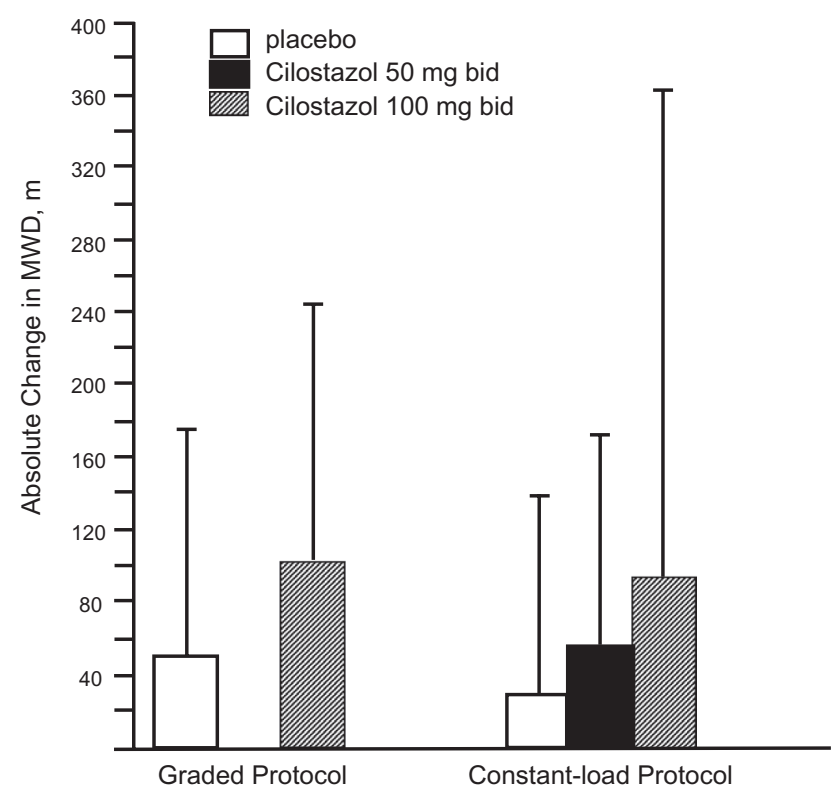

Figure 4 Mean absolute change in maximal walking distance (MWD), as measured by graded or constant-load protocols, among intermittent claudication patients receiving cilostazol $100 \mathrm{mg} 2 \times /$ day, cilostazol $50 \mathrm{mg} 2 \times /$ day, or placebo in six randomized controlled trials. Reprinted from Regensteiner JG,Ware JE Jr, McCarthy WJ, et al 2002. Effect of cilostazol on treadmill walking, community-based walking ability, and healthrelated quality of life in patients with intermittent claudication due to peripheral arterial disease: meta-analysis of six randomized controlled trials. J Am Geriatr Soc, 50: 1939-46. Copyright (C) 2002 with permission from Blackwell Publishing.
In a randomized, placebo-controlled study, patients receiving either cilostazol $100 \mathrm{mg} 2 \times /$ day, pentoxifylline $400 \mathrm{mg} 3 \times /$ day, or placebo were monitored for 24 weeks with cross-over to placebo after that period. When these patients were followed up for an additional 6 weeks, those in the cilostazol treatment group demonstrated a significant loss of treatment benefit after the change $(\mathrm{p}<0.05)$ (Cleanthis et al 2005), whereas there was no significant change following pentoxifylline therapy.

Interestingly, cilostazol has also been shown to provide long-term vessel patency after percutaneous transluminal angioplasty (PTA) in hemodialysis patients with PAD (Ishii et al 2008). In a recent Japanese study assessing lower extremity arterial patency rates 5 years after initial PTA, 193 hemodialysis patients with 372 consecutive lesions underwent successful PTA and were randomized to receive either cilostazol $100 \mathrm{mg} 2 \times$ /day in conjunction with standard medical treatment (71 patients with 130 lesions) or standard medical therapy without cilostazol (122 patients with 242 lesions). After 5 years, the patency rate was $52 \%$ in the cilostazol group and 33\% in the control group $(\mathrm{p}<0.05)$. Moreover, in the Cilostazol for Restenosis Trial (Douglas et al 2005), 750 patients with successful coronary bare metal stent implantation were randomized to receive either cilostazol $100 \mathrm{mg} 2 \times /$ day or placebo for 6 months in additional to aspirin and clopidogrel $75 \mathrm{mg}$ daily. At 6 months, the cilostazol group had a 36\% relative risk reduction in the restenosis rate compared with those who received placebo, as determined by quantitative coronary angiography. Furthermore, in the Cilostazol for Diabetic Patients in Drug-Eluting Stent Trial (Ahn et al 2008), 280 patients successfully underwent coronary stenting and were randomized to receive either aspirin and cilostazol or aspirin and clopidogrel. After a mean follow-up of 7.1 months, 237 patients underwent repeat coronary angiography; those randomized to receive aspirin and cilostazol had a $50 \%$ decrease in the incidence of restenosis compared to those who received aspirin and clopidogrel. The trial concluded that combination therapy with aspirin and cilostazol for the prevention of restenosis is comparable and may even be superior to that of aspirin and clopidogrel in diabetic patients who undergo drugeluting stent implantation.

Supervised exercise rehabilitation is an effective and safe intervention that has been demonstrated to improve the walking distance of patients with IC. Multiple controlled trials have demonstrated that both walking distance until the onset of claudication and absolute claudication distance 
improve in patients who receive supervised exercise rehabilitation. One meta-analysis of 21 studies found that patients who were treated with supervised exercise rehabilitation had a $180 \%$ increase in distance walked until the onset of claudication compared with a $40 \%$ increase in control group individuals. Similarly, the absolute distance walked increased by $130 \%$ in patients who received supervised exercise rehabilitation compared with a 30\% increase among those who did not (Gardner and Poehlman 1995). Another meta-analysis of 10 randomized trials comprising 250 patients with IC found that initial maximal walking time increased by approximately $150 \%$ in patients treated with exercise rehabilitation compared with control group individuals (Leng et al 2000). Overall, supervised exercise therapy has been shown to be very effective in improving maximal treadmill walking distance (Milani and Lavie 2007) (Figure 5).

\section{Conclusions}

In summary, cilostazol remains the only drug thus far proven to demonstrate consistent benefits in clinical trials in patients with IC. In addition to limb-specific outcomes, cilostazol has also been shown to provide pleotropic effects; these may provide additional clinical benefits, but clinical trials are needed to validate such effects. Finally, studies are also needed to compare the effects of cilostazol and supervised exercise training in patients with IC to either therapy by itself.

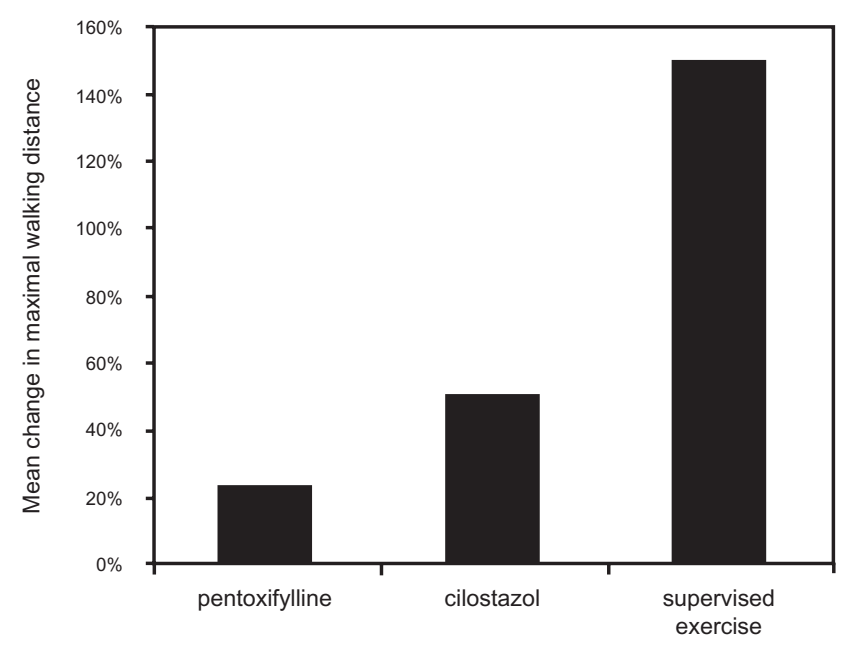

Figure 5 Mean improvement in maximal walking distance following medical interventions in patients with claudication. Reprinted from Milani RV, Lavie CJ. 2007. The role of exercise training in peripheral arterial disease. Vasc Med, 12:35I-8. Copyright (c) 2007 with permission from Sage Publishing.

\section{Disclosures}

None of the authors report conflicts of interest.

\section{References}

Agrawal NK, Maiti R, Dash D, et al. 2007. Cilostazol reduces inflammatory burden and oxidative stress in hypertensive type 2 diabetes mellitus patients. Pharmacol Res, 56:118-23.

Ahn Y, Jeong MH, Jeong JW, et al. 2008. Randomized comparison of cilostazol vs clopidogrel after drug-eluting stenting in diabetic patients - clilostazol for diabetic patients in drug-eluting stent (CIDES) trial. Circ J, 72:35-9.

Ainsworth BE, Haskell WL, Whitt MC, et al. 2000. Compendium of physical activities: an update of activity codes and MET intensities. Med Sci Sports Exerc, 32(Suppl):S498-504.

Aoki M, Morishita R, Hayashi S, et al. 2001. Inhibition of neointimal formation after balloon injury by cilostazol, accompanied by improvement of endothelial dysfunction and induction of hepatocyte growth factor in rat diabetes model. Diabetologia, 44:1034-42.

Beebe, HG, Dawson DL, Cutler BS, et al. 1999. A new pharmacological treatment for intermittent claudication: results of a randomized, multicenter trial. Arch Intern Med, 159:2041-50.

Bramer SL, Forbes WP, Mallikaarjun S. 1999. Cilostazol pharmacokinetics after single and multiple oral doses in healthy males and patients with intermittent claudication resulting from peripheral arterial disease. Clin Pharmacokinet, 37(Suppl 2):1-11.

Bramer SL, Tata PNV, Mallikaarjun S. 1997. Disposition of 14 C-cilostazol after single dose administration to healthy human subjects. Phar Res, 14(11 Suppl):S612.

Chapman, TM, Goa KL. 2003. Cilostazol: a review of its use in intermittent claudication. Am J Cardiovasc Drugs, 3:117-38.

Cleanthis M, Bhattacharya V, Smout J, et al. 2005. Combined aspirin and cilostazol treatment is associated with reduced platelet aggregation and prevention of exercise induced platelet activation. Yearb Soc Acad Res Surg, 39.

Dawson DL, Cutler BS, Meissner MH, et al. 1998. Cilostazol has beneficial effects in treatment of intermittent claudication: results from a multicenter, randomized, prospective, double-blind trial. Circulation, 98:678-86.

Dawson, DL, Cutler BS, Hiatt WR, et al. 2000. A comparison of cilostazol and pentoxifylline for treating intermittent claudication. $\mathrm{Am} \mathrm{J} \mathrm{Med,}$ 109:523-30.

Douglas JS Jr, Holmes DR Jr, Kereiakes DJ, et al. 2005. Cilostazol for Restenosis Trial (CREST) Investigators. Coronary stent restenosis in patients treated with cilostazol. Circulation, 112:2826-32.

Elam MB, Heckman J, Crouse JR, et al. 1998. Effect of the novel antiplatelet agent cilostazol on plasma lipoproteins in patients with intermittent claudication. Arterioscler Thromb Vasc Biol, 18:1942-7.

Gardner AW, Poehlman ET. 1995. Exercise rehabilitation programs for the treatment of claudication pain. A meta-analysis. JAMA, 274:975-80.

Hashimoto A, Miyakoda G, Hirose Y, et al. 2006. Activation of endothelial nitric oxide synthase by cilostazol via a cAMP/protein kinase A- and phosphatidylinositol 3-kinase/Akt-dependent mechanism. Atherosclerosis, 189:350-7.

Hayashi S, Morishita R, Matsushita H, et al. 2000. Cyclic AMP inhibited proliferation of human aortic vascular smooth muscle cells, accompanied by induction of $\mathrm{p} 53$ and $\mathrm{p} 21$. Hypertension, 35:237-43.

Hiatt WR, Wolfel EE, Regensteiner JG. 1992. Skeletal muscle carnitine metabolism in patients with unilateral peripheral arterial disease. J Appl Physiol, 73:346-53.

Hiatt WR. 2001. Medical treatment of peripheral arterial disease and claudication. N Engl J Med, 344:1608-21.

Hiatt WR. 2002. Pharmacologic therapy for peripheral arterial disease and claudication. J Vasc Surg, 36:1283-91.

Hiatt WR. 2006. The US experience with cilostazol in treating intermittent claudication. Atheroscler Suppl, 6:21-31. 
Hiatt, WR, Nawaz D, Brass EP. 1987. Carnitine metabolism during exercise in patients with peripheral vascular disease. J Appl Physiol, 62:2383-7.

Ikeda Y, Kikuchi M, Murakami H, et al. 1987. Comparison of the inhibitory effects of cilostazol, acetylsalicylic acid and ticlopidine on platelet functions ex vivo. Randomized, double-blind cross-over study. Arzneimittelforschung, 37:563-6.

Ishii H, Kumada Y, Toriyama T, et al. 2008. Cilostazol improves long-term patency after percutaneous transluminal angioplasty in hemodialysis patients with peripheral artery disease. Clin J Am Soc Nephrol, 3:1034-40.

Kumar M, Bhattacharya V. 2007. Cilostazol: a new drug in the treatment intermittent claudication. Recent Patents Cardiovasc Drug Discov, 2:181-5.

Lee TM, Su SF, Hwang JJ, et al. 2001. Differential lipogenic effects of cilostazol and pentoxifylline in patients with intermittent claudication: potential role for interleukin-6. Atherosclerosis, 158:471-6.

Leng GC, Fowler B, Ernst E. Exercise for intermittent claudication. 2000. Cochrane Database Syst Rev, 2:CD000990.

Manickavasagam S, Ye Y, Lin Y, et al. 2007. The cardioprotective effect of a statin and cilostazol combination: relationship to Akt and endothelial nitric oxide synthase activation. Cardiovasc Drugs Ther, 21:321-30.

Milani RV, Lavie CJ. 2007. The role of exercise training in peripheral arterial disease. Vasc Med, 12:351-8.

Money SR, Herd JA, Isaacsohn JL, et al. 1998. Effect of cilostazol on walking distances in patients with intermittent claudication caused by peripheral vascular disease. J Vasc Surg, 27:267-74; discussion 274-5.
Packer M, Carver JR, Rodeheffer RJ, et al. 1991. Effect of oral milrinone on mortality in severe chronic heart failure. The PROMISE Study Research Group. $N$ Engl J Med, 325:1468-75.

Pratt CM. 2001.Analysis of the cilostazol safety database. Am J Cardiol, 87:28D-33D.

Regensteiner JG, Ware JE Jr, McCarthy WJ, et al. 2002. Effect of cilostazol on treadmill walking, community-based walking ability, and healthrelated quality of life in patients with intermittent claudication due to peripheral arterial disease: meta-analysis of six randomized controlled trials. J Am Geriatr Soc, 50:1939-46.

Strandness DE, Dalman R, Panian S, et al. 1998. Two doses of cilostazol versus placebo in the treatment of claudication: results of a randomized, multicenter trial. Circulation, 98(17 Suppl 1):1-12.

Takahashi S, Oida K, Fujiwara R, et al. 1992. Effect of cilostazol, a cyclic AMP phosphodiesterase inhibitor, on the proliferation of rat aortic smooth muscle cells in culture. J Cardiovasc Pharmacol, 20:900-6.

Tamai Y, Takami H, Nakahata R, et al. 1999. Comparison of the effects of acetylsalicylic acid, ticlopidine and cilostazol on primary hemostasis using a quantitative bleeding time test apparatus. Haemostasis, 29:269-76.

Tani T, Sakurai K, Kimura Y, et al. 1992. Pharmacological manipulation of tissue cyclic AMP by inhibitors. Effects of phosphodiesterase inhibitors on the functions of platelets and vascular endothelial cells. Adv Second Messenger Phosphoprotein Res, 25:215-27. 
